\title{
A New Iterative Construction for Approximating Solutions of a Split Common Fixed Point Problem
}

\author{
Huimin He $\mathbb{D}^{1,2}$ Qinwei Fan, ${ }^{3}$ and Rudong Chen ${ }^{4}$ \\ ${ }^{1}$ School of Mathematics and Statistics, Xidian University, Xi'an 710071, China \\ ${ }^{2}$ School of Mathematics and Information Science, Guangzhou University, Guangzhou 510006, China \\ ${ }^{3}$ School of Science, Xi'an Polytechnic University, Xi'an 710048, China \\ ${ }^{4}$ Department of Mathematics, Tianjin Polytechnic University, Tianjin 300160, China \\ Correspondence should be addressed to Huimin He; huiminhe@126.com
}

Received 23 November 2020; Revised 27 December 2020; Accepted 11 January 2021; Published 30 January 2021

Academic Editor: Sun Young Cho

Copyright $\odot 2021$ Huimin He et al. This is an open access article distributed under the Creative Commons Attribution License, which permits unrestricted use, distribution, and reproduction in any medium, provided the original work is properly cited.

In this paper, we aim to construct a new strong convergence algorithm for a split common fixed point problem involving the demicontractive operators. It is proved that the vector sequence generated via the Halpern-like algorithm converges to a solution of the split common fixed point problem in norm. The main convergence results presented in this paper extend and improve some corresponding results announced recently. The highlights of this paper shed on the novel algorithm and the new analysis techniques.

\section{Introduction}

Let $H_{1}$ and $H_{2}$ be the Hilbert spaces and $C$ and $Q$ be nonempty closed and convex subsets of $H_{1}$ and $H_{2}$, respectively.

The split feasibility problem (SFP) is known to find

$$
x \in C, \quad \text { such that } A x \in Q \text {, }
$$

where $A: H_{1} \longrightarrow H_{2}$ is a linear bounded operator.

In [1], the split feasibility problem (SFP) in the finite-dimensional Hilbert spaces was introduced by Censor and Elfving. This problem is equivalent to a number of nonlinear optimization problems and finds numerous real applications, such as signal processing and medical imaging (see, e.g., [2-7]).

For this split problem, simultaneous multiprojections algorithm was employed by Censor and Elfving in the finitedimensional space $R^{n}$ to obtain the algorithm as follows:

$$
x_{n+1}=A^{-1} P_{Q} P_{A(C)} A x_{n},
$$

where both $C$ and $Q$ are convex and closed subsets of $R^{n}$, the linear bounded operator $A$ of $R^{n}$ is an $n \times n$ matrix, and $P_{Q}$ is the orthogonal projection operator onto the sets $Q$.
The above algorithm (2) involves the matrix $A^{-1}$ (one always assumes the existence of $A^{-1}$ ) at every iterative step. Calculating $A^{-1}$ is very much time-consuming, if the dimensions are large scale, in particular, and thus it does not become popular.

In order to overcome the fault, Byrne $[2,8]$ proposed the following novel algorithm $C Q$, which is under the spotlight of recent research

$$
x_{n+1}=P_{C}\left(x_{n}-\gamma A^{*}\left(I-P_{Q}\right) A x_{n}\right), \quad n \geq 0,
$$

where $P_{C}$ and $P_{Q}$ are the orthogonal projection operators onto the sets $C$ and $Q$, respectively, and $0<\gamma<(2 / \rho)$ with $\rho$ being the spectral radius of the composite mapping $A^{*} A$. But, the CQ algorithm's step-size is fixed, and it is related to spectral radius of $A^{*} A$. On the other hand, the orthogonal projection onto the subsets $C$ and $Q$ in Hilbert space $H_{1}$ is not easily calculated generally except the special cases, such as balls and polyhedrals. With the real applications (intensitymodulated radiation therapy and medical imaging) of the SFP in signal processing, the SFP has obtained much attention. Now, the approximate solutions of the SFP have been studied extensively by scholars and engineers (see, e.g., [9-13]). 
In (1), if $C$ and $Q$ are the intersections of fixed point sets of finite many nonlinear operators, the SFP becomes the split common fixed point problem (SCFPP). The SCFPP was studied first by Censor and Segal [14] in 2009, which consists of finding an element $x \in H_{1}$ with

$$
x \in \bigcap_{i=1}^{m} \operatorname{Fix}\left(T_{i}\right), \quad \text { s.t. } A x \in \bigcap_{j=1}^{n} \operatorname{Fix}\left(S_{j}\right),
$$

where Fix $\left(T_{i}\right)$ denotes the fixed point set of $T_{i}: H_{1} \longrightarrow H_{1}$ and Fix $\left(S_{j}\right)$ denotes the fixed point sets of $S_{i}: H_{2} \longrightarrow H_{2}$, respectively.

In particular, if $m=n=1$, then

$$
x \in \operatorname{Fix}(T), \quad \text { s.t. } A x \in \operatorname{Fix}(S),
$$

and $T: H_{1} \longrightarrow H_{1}, S: H_{2} \longrightarrow H_{2}$, and Fix $(T)$ denotes the fixed point set of $T$, and Fix $(S)$ denotes the fixed point set of $S$.

The SCFPP becomes a specific case of SFP and closely related to SFP. To solve this problem, the original algorithm for the directed operator was introduced by Censor and Segal [14] in 2009 as follows:

$$
x_{n+1}=T\left(x_{n}-\rho A^{*}(I-S) A x_{n}\right), \quad n \geq 0,
$$

where $\rho$ satisfies the constraint condition $0<\rho<\left(2 /\|A\|^{2}\right)$, and the authors got the weak convergence of the sequence $\left\{x_{n}\right\}$ for solving the SCFPP (5) if the SCFPP consists, that is, its solution set is nonempty.

Recently, Cui and Wang [15] studied the following algorithm, and they got the weak convergence of the sequence $\left\{x_{n}\right\}$ for solving the SCFPP (5):

$$
x_{n+1}=U_{\lambda}\left(x_{n}-\rho_{n} A^{*}(I-T) A x_{n}\right),
$$

where $U_{\lambda}=(1-\lambda) I+\lambda U$ and $\rho_{n}$ is given in the following pattern:

$$
\rho_{n}= \begin{cases}\frac{(1-\tau)\left\|(I-T) A x_{n}\right\|^{2}}{2\left\|A^{*}(I-T) A x_{n}\right\|^{2}}, & A x_{n} \neq T\left(A x_{n}\right), \\ 0, & \text { otherwise. }\end{cases}
$$

The step-size of this algorithm $\rho_{n}$ does not depend on the norm of the operator $A$ and searches automatically.

In 2015, Boikanyo [16] extended the main results of Cui and Wang [15] and constructed the Halpern-type algorithm for demicontractive operators that converge to a solution of the SCFPP (5) strongly:

$$
x_{n+1}=\alpha_{n} u+\left(1-\alpha_{n}\right) U_{\lambda}\left(x_{n}-\rho_{n} A^{*}(I-T) A x_{n}\right) \text {, }
$$

where $\rho_{n}$ is given as (8). In this result, the resolvent $I-$ $\rho_{n} A^{*}(I-T) A$ plays an important role. Indeed, the techniques of resolvents is quite popular, and it acts as a bridge between fixed point problems and a number of optimization problems (see, e.g., [17-21] and the references therein).

Motivated by the above results, we propose a novel algorithm on demicontractive operators for approximating a solution of the SCFPP (5):

$$
\left\{\begin{array}{l}
u_{n}=x_{n}-\rho_{n} A^{*}(I-T) A x_{n}, \\
x_{n+1}=\left(1-\alpha_{n}\right)\left\{\left(1-\xi_{n}\right) I+\xi_{n} U\left[\left(1-\eta_{n}\right) I+\eta_{n} U\right]\right\} u_{n}+\alpha_{n} u,
\end{array}\right.
$$

where $\rho_{n}$ is also obtained by (8). Our algorithm is also based on the Halpern iteration. Indeed, it is a core for many algorithms in split problems (see, e.g., [22-26]). We get the strong convergence of the iterative sequence $\left\{x_{n}\right\}$ generated by (10) for solving the SCFPP (5). Our main results are in two folds. First, we construct a novel iterative algorithm to solve the split common fixed point problem for the demicontractive operators. Second, we permit step-size to be selected self-adaptively by the self-adaptive method, which avoids to depend on the norm of the nonlinear operator $A$. Our results extend and improve some results of Boikanyo [16], Cui and Wang [15], Yao et al. [27], and many others.

\section{Preliminaries}

In this section, we will present some lemmas, which are useful to prove our main results as follows.

Let $H$ be a Hilbert space, which is endowed with the inner product $\langle\cdot, \cdot\rangle$, norm $\|\cdot\|$. Then, the following inequalities hold:

$$
\begin{array}{r}
\|u+v\|^{2} \leq\|u\|^{2}+2\langle v, u+v\rangle, \quad \forall u, v \in H, \\
\|t u+(1-t) v\|^{2}=t\|u\|^{2}+(1-t)\|v\|^{2}-t(1-t)\|u-v\|^{2}, \\
\forall t \in R \text { and } \forall u, v \in H .
\end{array}
$$

Definition 1. Let $T: H \longrightarrow H$ be an operator, then $I-T$ called demiclosed at zero, if the following implication holds for any $\left\{x_{n}\right\}$ in $H$ :

$$
\left.\begin{array}{ll}
x_{n} & -x \\
(I-T) x_{n} & \longrightarrow 0
\end{array}\right\} \Rightarrow x=T x .
$$

Note that the nonexpansive operator is demiclosed at zero [28].

Lemma 1 (see [29]). Let $\left\{a_{n}\right\}$ be a sequence of real nonnegative numbers with

$$
a_{n+1} \leq\left(1-\gamma_{n}\right) a_{n}+\delta_{n}
$$

where $\left\{\gamma_{n}\right\}$ is a sequence in $(0,1)$ and $\left\{\delta_{n}\right\}$ is a real sequence such that

(i) $\sum_{n=1}^{\infty} \gamma_{n}=\infty$

(ii) $\limsup _{n \longrightarrow \infty}\left(\delta_{n} / \gamma_{n}\right) \leq 0$ or $\sum_{n=1}^{\infty}\left|\delta_{n}\right|<\infty$

Then, $\lim _{n \longrightarrow \infty} a_{n}=0$.

Lemma 2 (see [15]). Let $A: H_{1} \longrightarrow H_{2}$ be a linear bounded operator and $\mathrm{T}: \mathrm{H}_{2} \longrightarrow \mathrm{H}_{2}$ a $\tau$-demicontractive mapping with $\tau<1$. If $A^{-1} \operatorname{Fix}(T) \neq \varnothing$, then it is as follows: 
(a) $(I-T) A \widehat{x}=0 \Leftrightarrow A^{*}(I-T) A \widehat{x}=0, \forall \hat{x} \in H_{1}$.

(b) In addition, for $z \in A^{-1} \operatorname{Fix}(T)$,

$$
\begin{aligned}
\| x & -z-\rho A^{*}(I-T) A \hat{x} \|^{2}+\frac{(1-\tau)^{2}\|(I-T) A \hat{x}\|^{4}}{4\left\|A^{*}(I-T) A \hat{x}\right\|^{2}} \\
& \leq\|\widehat{x}-z\|^{2},
\end{aligned}
$$

where $x \in H_{1}, A x \neq T(A x)$ and

$$
\rho:=\frac{(1-\tau)\|(I-T) A \widehat{x}\|^{2}}{2\left\|A^{*}(I-T) A \hat{x}\right\|^{2}} .
$$

Lemma 3 (see [30]). Let $H$ be a Hilbert space and let $T$ be an $L$-Lipschitzian mapping defined on $H$ with the module $L \geq 1$. Set

$$
K:=\xi T(\eta T+(1-\eta) I)+(1-\xi) I .
$$

If $0<\xi<\eta<\left(1 / 1+\sqrt{1+L^{2}}\right)$, then the following conclusions hold:

(1) $K$ is demiclosed at zero point 0 , if $T$ is demiclosed at 0

(2) $\operatorname{Fix}(T)=\operatorname{Fix}(T(\eta T+(1-\eta) I))=\operatorname{Fix}(K)$

(3) If $T: H \longrightarrow H$ is a quasi-pseudo-contractive operator, then the operator $K$ is quasi-non-expansive

Lemma 4 (see [31]). Let $\left\{s_{k}\right\}$ be a real numbers sequence that does not decrease at infinity in the sense that there exists a subsequence $\left\{s_{k_{j}}\right\}$ of $\left\{s_{k}\right\}$ such that $\left\{s_{k_{j}}\right\}<\left\{s_{k_{j+1}}\right\}$ for all $j \geq 0$. Define an integer sequence $\left\{m_{k}\right\}_{k \geq k_{0}}$ by

$$
m_{k}=\max \left\{k_{0} \leq l \leq k: s_{l}<s_{l+1}\right\} \text {. }
$$

Then, $m_{k} \longrightarrow \infty$ as $k \longrightarrow \infty$ and

$$
s_{m_{k}+1} \geq \max \left\{s_{m_{k}}, s_{k}\right\}
$$

for all $k \geq k_{0}$.

\section{Some Nonlinear Operators}

Definition 2. An operator $T: H \longrightarrow H$ is said to be $L$-Lipschitzian if and only if there exists $L>0$ such that

$$
\|T x-T y\| \leq L\|x-y\|,
$$

for all $x, y \in C$.

Definition 3. An operator $T: H \longrightarrow H$ is said to be nonexpansive if and only if

$$
\|T x-T y\| \leq\|x-z\|, \quad \forall x \in H .
$$

$$
\|T x-z\| \leq\|x-z\|, \quad \forall x \in H, \forall z \in \operatorname{Fix}(T) .
$$

Definition 5. An operator $T: H \longrightarrow H$ is said to be firmly nonexpansive if and only if

$$
\begin{array}{r}
\|T x-T y\|^{2} \leq\|x-y\|^{2}-\|(I-T) x-(I-T) y\|^{2}, \\
\forall x, y \in H .
\end{array}
$$

Definition 6. An operator $T: H \longrightarrow H$ is said to be firmly quasi-non-expansive if and only if $\operatorname{Fix}(T) \neq \varnothing$ and

$$
\|T x-z\|^{2} \leq\|x-z\|^{2}-\|(I-T) x\|^{2}, \quad \forall x \in H, \forall z \in \operatorname{Fix}(T) .
$$

Definition 7. An operator $T: H \longrightarrow H$ is said to be pseudocontractive if and only if

$$
\langle T x-T y, x-y\rangle \leq\|x-y\|^{2}, \quad \forall x, y \in H .
$$

Note that $T$ is pseudocontractive if and only if the operator $I-T$ is monotone. There is also an alternative definition for pseudocontractive operators, that is, $T$ is said to be pseudocontractive if and only if

$$
\begin{array}{r}
\|T x-T y\|^{2} \leq\|x-y\|^{2}+\|(I-T) x-(I-T) y\|^{2}, \\
\forall x, y \in H .
\end{array}
$$

Definition 8. An operator $T: H \longrightarrow H$ is said to be quasipseudo-contractive if and only if $\operatorname{Fix}(T) \neq \varnothing$ and

$$
\begin{array}{r}
\left\|T x-x^{*}\right\|^{2} \leq\left\|x-x^{*}\right\|^{2}+\|T x-x\|^{2}, \\
\forall x \in H, \forall x^{*} \in \operatorname{Fix}(T) .
\end{array}
$$

Definition 9. An operator $T: H \longrightarrow H$ is said to be strictly pseudocontractive if and only if there exists $k \in[0,1)$ such that

$$
\begin{array}{r}
\|T x-T y\|^{2} \leq\|x-y\|^{2}+k\|(I-T) x-(I-T) y\|^{2}, \\
\forall x, y \in H .
\end{array}
$$

Definition 10. A operator $T: H \longrightarrow H$ is said to be directed if and only if

$$
\langle z-T x, x-T x\rangle \leq 0, \quad \forall x \in H, \forall z \in \operatorname{Fix}(T) .
$$

Definition 11. An operator $T: H \longrightarrow H$ is said to be $\tau$-demicontractive with $\tau<1$ if and only if

$$
\begin{array}{r}
\|T x-z\|^{2} \leq\|x-z\|^{2}+\tau\|x-T x\|^{2}, \\
\forall x \in H, \forall z \in \operatorname{Fix}(T) .
\end{array}
$$

It is easy to obtain that (29) is equivalent to
Definition 4. An operator $T: H \longrightarrow H$ is said to be quasinon-expansive if and only if $\operatorname{Fix}(T) \neq \varnothing$ and 


$$
\begin{array}{r}
\|z-T x\|^{2}+\|x-T x\|^{2}-\|x-z\|^{2} \leq 0, \\
\forall x \in H, \forall z \in \operatorname{Fix}(T) .
\end{array}
$$

Remark 1. The classes of $k$-demicontrative mappings, directed mappings, quasi-non-expansive mappings, and nonexpansive mappings are closely related. By the above definitions, we obtain the following conclusion relations easily (see Figures 1-7).

(1) The nonexpansive mapping with $\operatorname{Fix}(T) \neq \varnothing$ is quasi-non-expansive mapping

(2) The quasi-non-expansive mapping is 0 -demicontrative mapping

(3) The firmly nonexpansive mapping is nonexpansive mapping

(4) The firmly quasi-non-expansive mapping is quasinon-expansive mapping

(5) The firmly nonexpansive mapping is firmly quasinon-expansive mapping

(6) The directed mapping is demicontractive mapping

(7) The demicontractive mapping is quasi-pseudocontractive mapping

(8) The strictly pseudocontractive mapping is pseudocontractive mapping

(9) The pseudocontractive mapping is quasi-pseudocontractive mapping

\section{Main Results}

In this section, some assumptions are as follows:

(1) $H_{1}$ and $H_{2}$ are two Hilbert spaces, $A: H_{1} \longrightarrow H_{2}$ is a linear bounded operator, and $A^{*}$ is the adjoint of $A$

(2) $U: H_{1} \longrightarrow H_{1}$ and $T: H_{2} \longrightarrow H_{2}$ are two $L$-Lipschitzian operators with $L \geq 1, \quad \operatorname{Fix}(U) \neq \varnothing$, $\operatorname{andFix}(T) \neq \varnothing$

(3) $U: H_{1} \longrightarrow H_{1}$ is a $\kappa$-demicontractive operator $(\kappa<1)$, and $T: H_{2} \longrightarrow H_{2}$ is a $\tau$-demicontractive operator $(\tau<1)$

(4) $I-U$ and $I-T$ are two demiclosed operators at $O$

(5) The set of solutions of SCFPP (5), denoted by $S$, is nonempty

The strong convergence of a sequence $\left\{x_{n}\right\}$ to a point $x \in H$ is denoted by $x_{n} \longrightarrow x$.

Now, we give the new algorithm to find $x^{*} \in S$.where $A$ is a bounded and linear mapping, $A^{*}$ is the adjoint of operator $A$, and $\rho_{n}$ is obtained as follows:

$$
\rho_{n}= \begin{cases}\frac{(1-\tau)\left\|(I-T) A x_{n}\right\|^{2}}{2\left\|A^{*}(I-T) A x_{n}\right\|^{2}}, & A x_{n} \neq T\left(A x_{n}\right), \\ 0, & \text { otherwise. }\end{cases}
$$

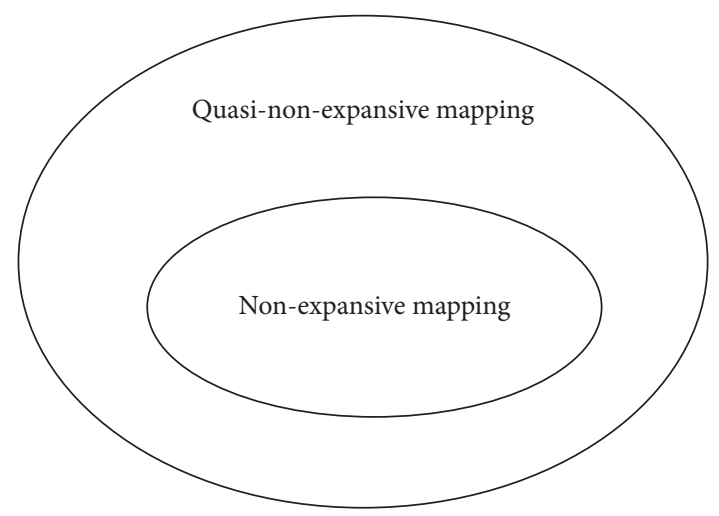

FIGURE 1: The relations of some nonlinear operators.

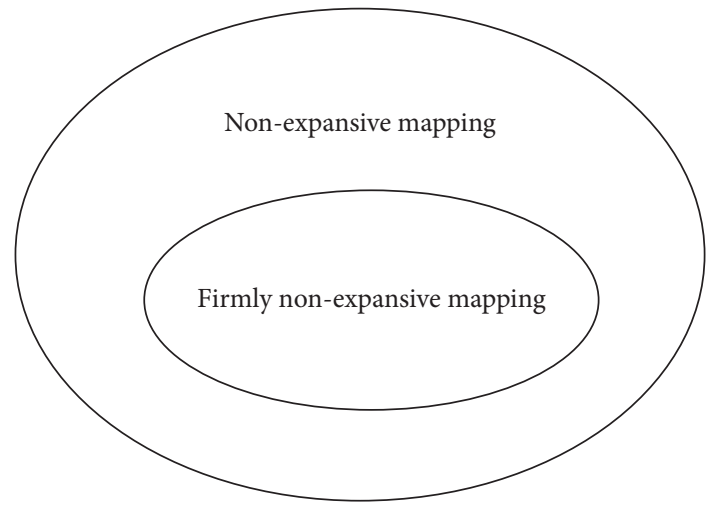

FIgURE 2: The relations of some nonlinear operators.

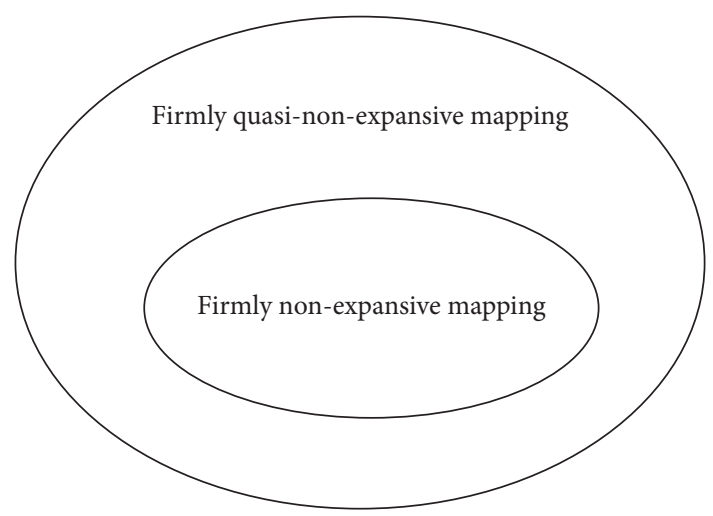

Figure 3: The relations of some nonlinear operators.

Algorithm 1. $H_{1}$ is a real Hilbert space, and $\operatorname{Fix}(U) \neq \varnothing$. Take an initial point $x_{0} \in H_{1}$ arbitrarily, and fix $u \in H_{1}$ and $\left\{\theta_{n}\right\} \subset(0,1)$. If the $n$-th iteration $x_{n}$ is available, then the $(n+1)$-th iteration is constructed via the following formula:

$$
\left\{\begin{array}{l}
u_{n}=x_{n}-\rho_{n} A^{*}(I-T) A x_{n}, \\
x_{n+1}=\theta_{n} u+\left(1-\theta_{n}\right)\left\{\left(1-\mu_{n}\right) I+\mu_{n} U\left[\left(1-v_{n}\right) I+v_{n} U\right]\right\} u_{n},
\end{array}\right.
$$

Lemma 5. Assume that $H_{1}$ is a Hilbert space, $U: H_{1} \longrightarrow H_{1}$ is a $\kappa$-demicontractive operator with $\kappa \leq 1$, L-Lipschitzian 


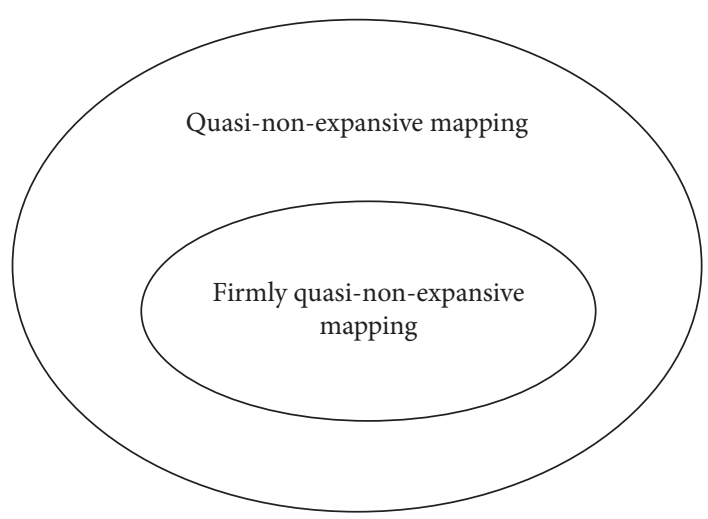

FIgURE 4: The relations of some nonlinear operators.

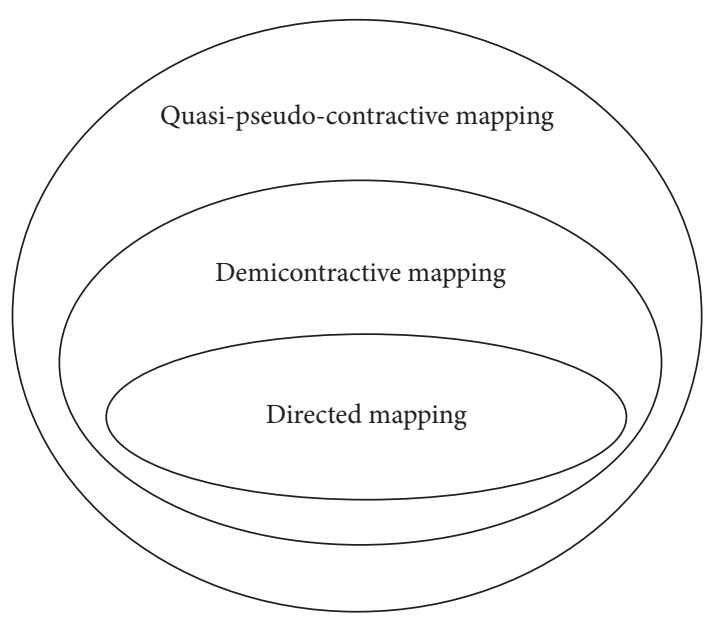

Figure 5: The relations of some nonlinear operators.

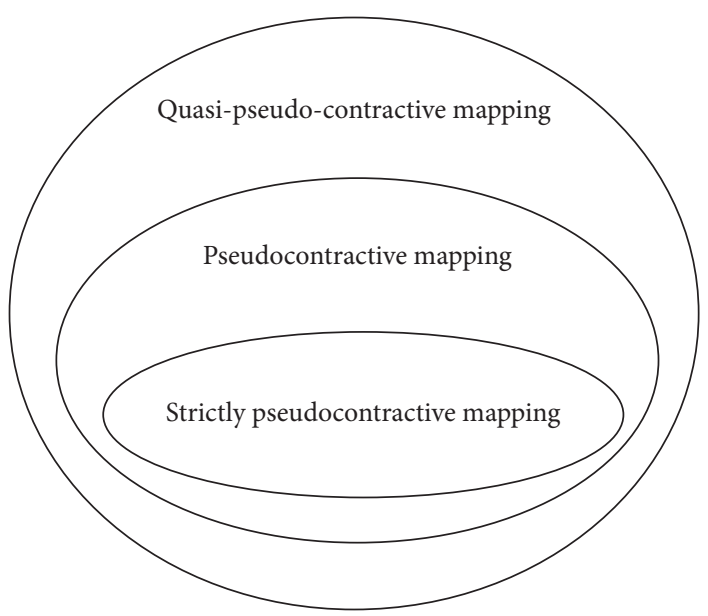

Figure 6: The relations of some nonlinear operators.

mappings $(L \geq 1)$, and Fix $(U) \neq \varnothing$. Denote $U_{\mu, \nu}:=(1-\mu) I+$ $\mu U[(1-v) I+\nu U] \quad$ with $\quad 0<\mu<\nu<(2-\kappa / 1+$ $\left.\sqrt{1+L^{2}(2-\kappa)}\right)$. Then, for all $x \in H_{1}$,

$$
\left\|z-U_{\mu, \nu}\right\|^{2} \leq\|x-z\|^{2}-\mu \nu\left(2-2 \nu-\kappa-\nu^{2} L^{2}\right)\|U x-x\|^{2},
$$

where $z \in$ Fix $(U)$. Moreover,

$$
\left\|z-U_{\mu, \nu}\right\| \leq\|z-x\| .
$$

That is, $U_{\mu, \nu}$ is quasi-non-expansive.

Proof. Since $z \in \operatorname{Fix}(U)$, we get from (30) that 


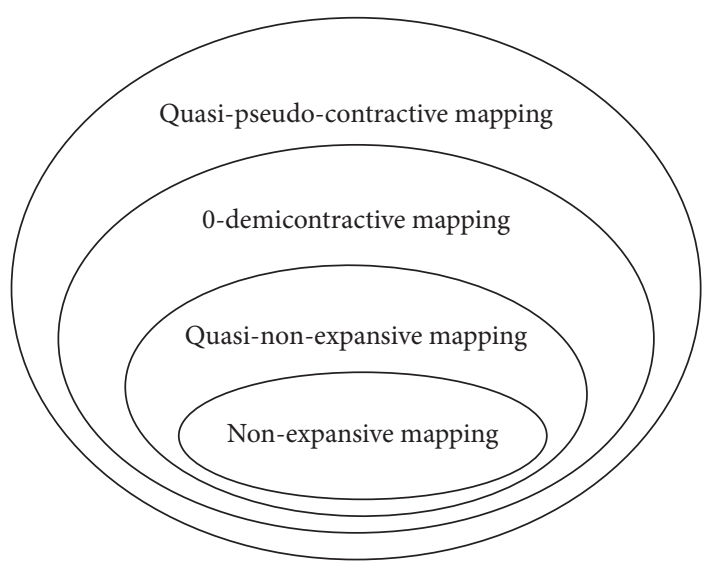

Figure 7: The relations of some nonlinear operators.

$$
\begin{aligned}
\| U & {[(1-v) I+\nu U] x-z \|^{2} } \\
\leq & \|[(1-v) I+\nu U] x-z\|^{2} \\
& +\kappa\|[(1-v) I+\nu U] x-U[(1-v) I+\nu U] x\|^{2} \\
\leq & \|(1-v)(x-z)+\nu(U x-z)\|^{2} \\
& +\kappa\|[(1-v) I+\nu U] x-U[(1-\nu) I+\nu U] x\|^{2} .
\end{aligned}
$$

Based on the fact that $U$ is $L$-Lipschitzian, we get

$$
\|U x-U[(1-\nu) I+\nu U] x\| \leq \nu L\|x-U x\| .
$$

Also, from (30) and (12), we can get

$$
\begin{aligned}
&\|(1-\nu)(x-z)+\nu(U x-z)\|^{2} \\
&=(1-v)\|x-z\|^{2}+\nu\|U x-z\|^{2}-\nu(1-v)\|x-U x\|^{2} \\
& \leq(1-v)\|x-z\|^{2}+\nu\left(\|x-z\|^{2}+\kappa\|U x-x\|^{2}\right) \\
& \quad-v(1-v)\|x-U x\|^{2} \\
&=\|x-z\|^{2}+\nu(\nu+\kappa-1)\|U x-x\|^{2} .
\end{aligned}
$$

By (12) and (37), we get

$$
\begin{aligned}
& \|[(1-\nu) I+\nu U] x-U[(1-\nu) I+\nu U] x\|^{2} \\
& =\|(1-v)(x-U[(1-v) I+\nu U] x)+\nu(U x-U[(1-v) I+\nu U] x)\|^{2} \\
& =(1-v)\|x-U[(1-v) I+\nu U] x\|^{2}+\nu\|U x-U[(1-\nu) I+\nu U] x\|^{2} \\
& -v(1-v)\|x-U x\|^{2} \\
& \leq(1-v)\|x-U[(1-\nu) I+\nu U] x\|^{2}+\nu \nu^{2} L^{2}\|U x-x\|^{2} \\
& -v(1-v)\|x-U x\|^{2} \\
& =(1-v)\|x-U[(1-v) I+\nu U] x\|^{2}-\nu\left(1-v-v^{2} L^{2}\right)\|x-U x\|^{2} .
\end{aligned}
$$


Substituting (38) and (39) into (36), we have

$$
\begin{aligned}
\| U[( & 1-\nu) I+\nu U] x-z \|^{2} \\
\leq & \|x-z\|^{2}+\nu(\nu+\kappa-1)\|U x-x\|^{2} \\
& +(1-v)\|x-U[(1-v) I+\nu U] x\|^{2} \\
& -v\left(1-v-v^{2} L^{2}\right)\|x-U x\|^{2} \\
= & \|x-z\|^{2}+(1-v)\|x-U[(1-\nu) I+\nu U] x\|^{2} \\
& -v\left(2-2 \nu-\kappa-\nu^{2} L^{2}\right)\|x-U x\|^{2} .
\end{aligned}
$$

Since $\mu<v$, combining (12) and (40), we get

$$
\begin{aligned}
& \|(1-\mu) x+\mu U[(1-\nu) I+\nu U] x-z\|^{2} \\
& =\|(1-\mu)(x-z)+\mu\{U[(1-\nu) I+\nu U] x-z\}\} \|^{2} \\
& =(1-\mu)\|x-z\|^{2}+\mu\|U[(1-\nu) I+\nu U] x-z\|^{2} \\
& -\mu(1-\mu)\|U[(1-\nu) I+\nu U] x-x\|^{2} \\
& =(1-\mu)\|x-z\|^{2}-\mu(1-\mu)\|U[(1-\nu) I+\nu U] x-x\|^{2} \\
& +\mu\left[\|x-z\|^{2}+(1-v)\|x-U[(1-\nu) I+\nu U] x\|^{2}\right. \\
& -v\left(2-2 v-\kappa-v^{2} L^{2}\right)\|x-U x\|^{2} \\
& =\|x-z\|^{2}+\mu(\mu-\nu)\|x-U[(1-\nu) I+\nu U] x\|^{2} \\
& -v\left(2-2 v-\kappa-v^{2} L^{2}\right)\|x-U x\|^{2} \\
& \leq\|x-z\|^{2}-v\left(2-2 \nu-\kappa-v^{2} L^{2}\right)\|x-U x\|^{2} .
\end{aligned}
$$

Since $v<\left(2-\kappa / 1+\sqrt{1+L^{2}(2-\kappa)}\right)$, we deduce

$$
2-2 v-\kappa-v^{2} L^{2}>0 \text {. }
$$

Hence,

$\|(1-\mu) x+\mu U[(1-v) I+\nu U] x-z\|^{2} \leq\|x-z\|^{2}$.

That is, $U_{\mu, \nu}$ is quasi-non-expansive.

Theorem 1. Assume that problem (5) is consistent $(S \neq \varnothing)$. Let $H_{1}, H_{2}, A, U, T,\left\{x_{n}\right\}$ be the same as above. If $\theta_{n} \subset(0,1)$ satisfies $\lim _{n \longrightarrow \infty} \theta_{n}=0$ and $\sum_{n=0}^{\infty} \theta_{n}=\infty$, where $a$ and $b$ are constants and $\left\{\mu_{n}\right\}$ and $\left\{v_{n}\right\}$ satisfies $0<a<\mu_{n}<\nu_{n}<$ $b<\left(2-\kappa / 1+\sqrt{1+L^{2}(2-\kappa)}\right), \forall n \geq 1$, then the sequence $\left\{x_{n}\right\}$ converges to a point $\bar{x} \in S$ in norm and $\bar{x}$ is the nearest point $S$ to $u\left(\bar{x}=t P_{S} n u\right)$.

Proof. This proof is split into three parts as follows.

Step 1. Prove that $\left\{x_{n}\right\}$ is a bounded sequence.

Take $p \in S$. From Theorem 1 , we know that $U_{\mu_{n}, v_{n}}$ is quasi-non-expansive. From (32), we have

$$
\begin{aligned}
\left\|x_{n+1}-p\right\| & =\left\|\theta_{n} u+\left(1-\theta_{n}\right) U_{\mu_{n}, v_{n}} u_{n}-p\right\| \\
& =\left\|\theta_{n}(u-p)+\left(1-\theta_{n}\right)\left(U_{\mu_{n}, v_{n}} u_{n}-p\right)\right\| \\
& \leq \theta_{n}\|u-p\|+\left(1-\theta_{n}\right)\left\|U_{\mu_{n}, v_{n}} u_{n}-p\right\| \\
& \leq \theta_{n}\|u-p\|+\left(1-\theta_{n}\right)\left\|u_{n}-p\right\| \\
& \leq \theta_{n}\|u-p\|+\left(1-\theta_{n}\right)\left\|x_{n}-p\right\| .
\end{aligned}
$$

By induction, we get

$$
\left\|x_{n}-p\right\| \leq \max \left\{\|u-p\|,\left\|x_{0}-p\right\|\right\} .
$$

Thus, $\left\{x_{n}\right\}$ is bounded.

Step 2

$$
\left\|x_{n+1}-\bar{x}\right\|^{2} \leq\left(1-\theta_{n}\right)\left\|x_{n}-\bar{x}\right\|^{2}+2 \theta_{n}\left\langle u-\bar{x}, x_{n+1}-\bar{x}\right\rangle,
$$

where $\bar{x}=P_{S} u$.

Consider the case $\rho_{n} \neq 0$. From (32), (35), and (11), we get

$$
\begin{aligned}
\left\|x_{n+1}-\bar{x}\right\|^{2}= & \left\|\theta_{n} u+\left(1-\theta_{n}\right) U_{\mu_{n}, \nu_{n}} u_{n}-\bar{x}\right\| \\
= & \left\|\theta_{n}(u-\bar{x})+\left(1-\theta_{n}\right)\left(U_{\mu_{n}, v_{n}} u_{n}-\bar{x}\right)\right\| \\
\leq & \left(1-\theta_{n}\right)^{2}\left\|U_{\mu_{n}, \nu_{n}} u_{n}-\bar{x}\right\|^{2}+2 \theta_{n}\left\langle u-\bar{x}, x_{n+1}-\bar{x}\right\rangle \\
\leq & \left(1-\theta_{n}\right)\left\|U_{\mu_{n}, v_{n}} u_{n}-\bar{x}\right\|^{2}+2 \theta_{n}\left\langle u-\bar{x}, x_{n+1}-\bar{x}\right\rangle \\
\leq & \left(1-\theta_{n}\right)\left\|u_{n}-\bar{x}\right\|^{2}+2 \theta_{n}\left\langle u-\bar{x}, x_{n+1}-\bar{x}\right\rangle \\
\leq & \left(1-\theta_{n}\right)\left[\left\|x_{n}-\bar{x}\right\|^{2}-\frac{(1-\tau)^{2}}{4} \frac{\left\|(I-T) A x_{n}\right\|^{4}}{\left\|A^{*}(I-T) A x_{n}\right\|^{2}}\right] \\
& +2 \theta_{n}\left\langle u-\bar{x}, x_{n+1}-\bar{x}\right\rangle \\
\leq & \left(1-\theta_{n}\right)\left\|x_{n}-\bar{x}\right\|^{2}+2 \theta_{n}\left\langle u-\bar{x}, x_{n+1}-\bar{x}\right\rangle .
\end{aligned}
$$

Hence,

$$
\left\|x_{n+1}-\bar{x}\right\|^{2} \leq\left(1-\theta_{n}\right)\left\|x_{n}-\bar{x}\right\|^{2}+2 \theta_{n}\left\langle u-\bar{x}, x_{n+1}-\bar{x}\right\rangle .
$$

Consider the case $\rho_{n}=0$. From (32) and (11), we get

$$
\begin{aligned}
\left\|x_{n+1}-\bar{x}\right\|^{2} & =\left\|\theta_{n} u+\left(1-\theta_{n}\right) U_{\mu_{n}, v_{n}} u_{n}-\bar{x}\right\| \\
& =\left\|\theta_{n}(u-\bar{x})+\left(1-\theta_{n}\right)\left(U_{\mu_{n}, v_{n}} u_{n}-\bar{x}\right)\right\| \\
& \leq\left(1-\theta_{n}\right)^{2}\left\|U_{\mu_{n}, v_{n}} u_{n}-\bar{x}\right\|^{2}+2 \theta_{n}\left\langle u-\bar{x}, x_{n+1}-\bar{x}\right\rangle \\
& \leq\left(1-\theta_{n}\right)\left\|U_{\mu_{n}, v_{n}} u_{n}-\bar{x}\right\|^{2}+2 \theta_{n}\left\langle u-\bar{x}, x_{n+1}-\bar{x}\right\rangle \\
& \leq\left(1-\theta_{n}\right)\left\|u_{n}-\bar{x}\right\|^{2}+2 \theta_{n}\left\langle u-\bar{x}, x_{n+1}-\bar{x}\right\rangle \\
& \leq\left(1-\theta_{n}\right)\left\|x_{n}-\bar{x}\right\|^{2}+2 \theta_{n}\left\langle u-\bar{x}, x_{n+1}-\bar{x}\right\rangle .
\end{aligned}
$$


Hence,

$\left\|x_{n+1}-\bar{x}\right\|^{2} \leq\left(1-\theta_{n}\right)\left\|x_{n}-\bar{x}\right\|^{2}+2 \theta_{n}\left\langle u-\bar{x}, x_{n+1}-\bar{x}\right\rangle$.

Step 3. Prove that $x_{n} \longrightarrow \bar{x}$ as $n \longrightarrow \infty$.

This step is divided into two cases. Denote $s_{n}:=\left\|x_{n}-\bar{x}\right\|^{2}$.

Case 1. Assume there exists a positive integer $n_{0}$ and the sequence $\left\{s_{n}\right\}$ is decreasing for any $n \geq n_{0}$. Then, $\left\{s_{n}\right\}$ converges to some point strongly by the monotonic bounded principle.

First, we show that

$$
\underset{n \rightarrow \infty}{\limsup }\left\langle u-\bar{x}, x_{n}-\bar{x}\right\rangle \leq 0 .
$$

Using the choice (33) of the step-size $\rho_{n},(32),(34),(35)$, and (11), we get

$$
\begin{aligned}
\left\|x_{n+1}-\bar{x}\right\|^{2}= & \left\|\theta_{n} u+\left(1-\theta_{n}\right) U_{\mu_{n}, v_{n}} u_{n}-\bar{x}\right\| \\
& =\left\|\theta_{n}(u-\bar{x})+\left(1-\theta_{n}\right)\left(U_{\mu_{n}, v_{n}} u_{n}-\bar{x}\right)\right\| \\
\leq & \left(1-\theta_{n}\right)^{2}\left\|U_{\mu_{n}, \nu_{n}} u_{n}-\bar{x}\right\|^{2}+2 \theta_{n}\left\langle u-\bar{x}, x_{n+1}-\bar{x}\right\rangle \\
\leq & \left\|U_{\mu_{n}, v_{n}} u_{n}-\bar{x}\right\|^{2}+2 \theta_{n}\left\langle u-\bar{x}, x_{n+1}-\bar{x}\right\rangle \\
\leq & \left\|u_{n}-\bar{x}\right\|^{2}-\mu_{n} \nu_{n}\left(2-2 v_{n}-\kappa-v_{n}^{2} L^{2}\right)\left\|U u_{n}-u_{n}\right\|^{2} \\
& +2 \theta_{n}\left\langle u-\bar{x}, x_{n+1}-x\right\rangle \\
\leq & \left\|x_{n}-\bar{x}\right\|^{2}-\frac{\left\|(I-T) A x_{n}\right\|^{4}}{\left\|A^{*}(I-T) A x_{n}\right\|^{2}} \frac{(1-\tau)^{2}}{4} \\
& -\mu_{n} v_{n}\left(2-2 v_{n}-\kappa-v_{n}^{2} L^{2}\right)\left\|U u_{n}-u_{n}\right\|^{2} \\
& +2 \theta_{n}\left\langle u-\bar{x}, x_{n+1}-\bar{x}\right\rangle .
\end{aligned}
$$

So,

$$
\begin{aligned}
& \mu_{n} v_{n}\left(2-2 v_{n}-\kappa-v_{n}^{2} L^{2}\right)\left\|U u_{n}-u_{n}\right\|^{2} \leq s_{n}-s_{n+1}+\theta_{n} L, \\
& 0 \leq \frac{(1-\tau)^{2}\left\|(I-T) A x_{n}\right\|^{4}}{4\left\|A^{*}(I-T) A x_{n}\right\|^{2}} \leq s_{n}-s_{n+1}+\theta_{n} L,
\end{aligned}
$$

where $L$ is a nonnegative real constant such that $\sup _{n \in N}\left\{2\left\langle f\left(x_{n}\right)-\bar{x}, x_{n+1}-\bar{x}\right\rangle\right\} \leq L$. Based on the fact that $\left\{s_{n}\right\}$ is convergent, we have

$$
\left\|u_{n}-U u_{n}\right\| \longrightarrow 0, \quad \text { as } n \longrightarrow \infty,
$$

$$
\frac{\left\|(I-T) A x_{n}\right\|^{2}}{\left\|A^{*}(I-T) A x_{n}\right\|} \longrightarrow 0, \quad \text { as } n \longrightarrow \infty .
$$

Moreover,

$\frac{\left\|(I-T) A x_{n}\right\|^{2}}{\left\|A^{*}(I-T) A x_{n}\right\|} \geq \frac{\left\|(I-T) A x_{n}\right\|^{2}}{\left\|(I-T) A x_{n}\right\| \cdot\|A\|} \geq \frac{\left\|(I-T) A x_{n}\right\|}{\|A\|}$.

Hence,

$$
\left\|A x_{n}-T A x_{n}\right\| \longrightarrow 0
$$

Since

$$
\begin{aligned}
\left\|x_{n}-u_{n}\right\| & =\rho_{n}\left\|A^{*}(I-T) A x_{n}\right\| \\
& =\frac{(1-\tau)\left\|(I-T) A x_{n}\right\|^{2}}{2\left\|A^{*}(I-T) A x_{n}\right\|} \longrightarrow 0, \text { as } n \longrightarrow \infty .
\end{aligned}
$$

Since $x_{n} \rightarrow q$, we have $u_{n} \rightarrow q$ due to (58). From (54) and as $I-U$ is demiclosed at zero, we have

$$
q \in \operatorname{Fix}(U) \text {. }
$$

From (55) and $I-T$ is demiclosed at zero, we have

$$
A q \in \operatorname{Fix}(T) \text {. }
$$

Thus, $q \in S$ by (59) and (60). Hence, it follows from $\bar{x}=$ $P_{S} u$ that

$$
\begin{aligned}
\text { limsup } \backslash \text { limits_n } & \longrightarrow \infty\left\langle u-\bar{x}, x_{n}-\bar{x}\right\rangle \\
& =\langle u-\bar{x}, q-\bar{x}\rangle \leq 0 .
\end{aligned}
$$

Secondly, we show that

$$
\left\|x_{n+1}-x_{n}\right\| \longrightarrow 0, \quad \text { as } n \longrightarrow \infty .
$$

From (32), we have

$$
\begin{aligned}
\left\|U_{\mu_{n}, v_{n}} u_{n}-u_{n}\right\| & =\mu_{n}\left\|u_{n}-U\left[\left(1-v_{n}\right) I+v_{n} U\right] u_{n}\right\| \\
& =\mu_{n}\left\|u_{n}-U u_{n}+U u_{n}-U\left[\left(1-v_{n}\right) I+v_{n} U\right] u_{n}\right\| \\
& \leq \mu_{n}\left\|u_{n}-U u_{n}\right\|+\mu_{n}\left\|U u_{n}-U\left[\left(1-v_{n}\right) I+v_{n} U\right] u_{n}\right\| \\
& \leq \mu_{n}\left\|u_{n}-U u_{n}\right\|+\mu_{n} L\left\|u_{n}-\left[\left(1-v_{n}\right) I+v_{n} U\right] u_{n}\right\| \\
& =\mu_{n}\left\|u_{n}-U u_{n}\right\|+\mu_{n} v_{n} L\left\|u_{n}-U u_{n}\right\| \\
& =\mu_{n}\left(1+v_{n} L\right)\left\|u_{n}-U u_{n}\right\| .
\end{aligned}
$$

From the above equation and (32), (54), and (58), we have 


$$
\begin{aligned}
\left\|x_{n+1}-x_{n}\right\| & \leq \theta_{n}\left\|u-x_{n}\right\|+\left(1-\theta_{n}\right)\left\|x_{n}-U_{\mu_{n}, v_{n}} u_{n}\right\| \\
& \leq \theta_{n}\left\|u-x_{n}\right\|+\left\|x_{n}-u_{n}\right\|+\left\|u_{n}-U_{\mu_{n}, v_{n}} u_{n}\right\| \\
& \leq \theta_{n}\left\|u-x_{n}\right\|+\left\|x_{n}-u_{n}\right\|+\mu_{n}\left(1+v_{n} L\right)\left\|u_{n}-U u_{n}\right\| \\
& \leq \theta_{n}\left\|u-x_{n}\right\|+\left\|x_{n}-u_{n}\right\|+b(1+b L)\left\|u_{n}-U u_{n}\right\| .
\end{aligned}
$$

Combining (54) and 58, we get

$$
\left\|x_{n+1}-x_{n}\right\| \longrightarrow 0, \quad \text { as } n \longrightarrow \infty .
$$

Thirdly, we show that $x_{n} \longrightarrow \bar{x}$ as $n \longrightarrow \infty$.

Together with (51) and (62), we get

$$
\limsup _{n \longrightarrow \infty}\left\langle u-\bar{x}, x_{n+1}-\bar{x}\right\rangle \leq 0 .
$$

Applying Lemma 2 to (46), which together with the assumption of $\left\{\theta_{n}\right\}$ and (66), we get $x_{n} \longrightarrow \bar{x}$ as $n \longrightarrow \infty$ easily.

Case 2. Assume that there is no positive integer $n_{0}$ and a decreasing sequence $\left\{s_{n}\right\}$ for any $n \geq n_{0}$. That is, there is a subsequence $\left\{s_{k_{i}}\right\}$ of $\left\{s_{k}\right\}$ such that $s_{k_{i}}<s_{k_{i}+1}$ for any $i \in N$.

From Lemma 4, we can define a nondecreasing sequence $\left\{m_{k}\right\} \subset N$ such that $m_{k} \longrightarrow \infty$ as $k \longrightarrow \infty$ and

$$
s_{m_{k}} \leq s_{m_{k}+1} \text {. }
$$

Firstly, we show

$$
\underset{n \longrightarrow \infty}{\limsup }\left\langle u-\bar{x}, x_{m_{k}}-\bar{x}\right\rangle \leq 0 .
$$

It follows from (52) and (67) and the boundedness of $\left\{x_{m_{k}}\right\}$ that

$$
\begin{aligned}
\mu_{m_{k}} v_{m_{k}}\left(2-2 v_{m_{k}}-\kappa-v_{m_{k}}^{2} L^{2}\right)\left\|U u_{m_{k}}-u_{m_{k}}\right\|^{2} & \leq s_{m_{k}}-s_{m_{k}+1}+\alpha_{m_{k}} L \\
& \leq \alpha_{m_{k}} L, \\
0 \leq \frac{(1-\tau)^{2}}{4} \frac{\left\|(I-T) A x_{m_{k}}\right\|^{4}}{\left\|A^{*}(I-T) A x_{m_{k}}\right\|^{2}} & \leq s_{m_{k}}-s_{m_{k}+1}+\alpha_{m_{k}} L \\
& \leq \alpha_{m_{k}} L .
\end{aligned}
$$

Thus,

$$
\begin{gathered}
\left\|u_{m_{k}}-U u_{m_{k}}\right\| \longrightarrow 0, \quad \text { as } n \longrightarrow \infty, \\
\frac{\left\|(I-T) A x_{m_{k}}\right\|^{2}}{\left\|A^{*}(I-T) A x_{m_{k}}\right\|} \longrightarrow 0, \quad \text { as } n \longrightarrow \infty .
\end{gathered}
$$

Moreover

$$
\frac{1}{\|A\|}\left\|(I-T) A x_{m_{k}}\right\| \leq \frac{\left\|(I-T) A x_{m_{k}}\right\|^{2}}{\|A\| \cdot\left\|(I-T) A x_{m_{k}}\right\|} \leq \frac{\left\|(I-T) A x_{m_{k}}\right\|^{2}}{\left\|A^{*}(I-T) A x_{m_{k}}\right\|^{2}} .
$$

Hence,

$$
\left\|A x_{m_{k}}-T A x_{m_{k}}\right\| \longrightarrow 0
$$

due to

$$
\begin{aligned}
\left\|x_{m_{k}}-u_{m_{k}}\right\| & =\rho_{m_{k}}\left\|A^{*}(I-T) A x_{m_{k}}\right\| \\
& =\frac{(1-\tau)\left\|(I-T) A x_{m_{k}}\right\|^{2}}{2\left\|A^{*}(I-T) A x_{m_{k}}\right\|} \longrightarrow 0, \quad \text { as } n \longrightarrow \infty .
\end{aligned}
$$

Since $x_{m_{k}} \rightarrow q$, then $u_{m_{k}} \rightarrow q$. So, we have $q \in S$ by the similar proofs in Case 1. Hence, it follows from $\bar{x}=P_{S} u$ that

$$
\underset{n \longrightarrow \infty}{\limsup }\left\langle u-\bar{x}, x_{m_{k}}-\bar{x}\right\rangle=\langle u-\bar{x}, q-\bar{x}\rangle \leq 0 .
$$

Secondly, we show

$$
\left\|x_{m_{k}+1}-x_{m_{k}}\right\| \longrightarrow 0, \quad \text { as } k \longrightarrow \infty .
$$

From (32), we have

$$
\begin{aligned}
& \left\|U_{\mu_{m_{k}}, v_{m_{k}}} u_{m_{k}}-u_{m_{k}}\right\| \\
& \quad=\mu_{m_{k}}\left\|u_{m_{k}}-U\left[\left(1-v_{m_{k}}\right) I+v_{m_{k}} U\right] u_{m_{k}}\right\| \\
& \quad=\mu_{m_{k}}\left\|u_{m_{k}}-U u_{m_{k}}+U u_{m_{k}}-U\left[\left(1-v_{m_{k}}\right) I+v_{m_{k}} U\right] u_{m_{k}}\right\| \\
& \leq \mu_{m_{k}}\left\|u_{m_{k}}-U u_{m_{k}}\right\|+\mu_{m_{k}}\left\|U u_{m_{k}}-U\left[\left(1-v_{m_{k}}\right) I+v_{m_{k}} U\right] u_{m_{k}}\right\| \\
& \leq \mu_{m_{k}}\left\|u_{m_{k}}-U u_{m_{k}}\right\|+\mu_{m_{k}} L\left\|u_{m_{k}}-\left[\left(1-v_{m_{k}}\right) I+v_{m_{k}} U\right] u_{m_{k}}\right\| \\
& =\mu_{m_{k}}\left\|u_{m_{k}}-U u_{m_{k}}\right\|+\mu_{m_{k}} v_{m_{k}} L\left\|u_{m_{k}}-U u_{m_{k}}\right\| \\
& =\mu_{m_{k}}\left(1+v_{m_{k}} L\right)\left\|u_{m_{k}}-U u_{m_{k}}\right\| .
\end{aligned}
$$

By the above equation and (32), we have 


$$
\begin{aligned}
& \left\|x_{m_{k}+1}-x_{m_{k}}\right\| \\
& \quad \leq \alpha_{m_{k}}\left\|u-x_{m_{k}}\right\|+\left(1-\alpha_{m_{k}}\right)\left\|x_{m_{k}}-U_{\mu_{m_{k}}, v_{m_{k}}} u_{m_{k}}\right\| \\
& \quad \leq \alpha_{m_{k}}\left\|u-x_{m_{k}}\right\|+\left\|x_{m_{k}}-u_{m_{k}}\right\|+\left\|u_{m_{k}}-U_{\mu_{m_{k}}, v_{m_{k}}} u_{m_{k}}\right\| \\
& \leq \alpha_{m_{k}}\left\|u-x_{m_{k}}\right\|+\left\|x_{m_{k}}-u_{m_{k}}\right\|+\mu_{m_{k}}\left(1+v_{m_{k}} L\right)\left\|u_{m_{k}}-U u_{m_{k}}\right\| \\
& \quad \leq \alpha_{m_{k}}\left\|u-x_{m_{k}}\right\|+\left\|x_{m_{k}}-u_{m_{k}}\right\|+b(1+b L)\left\|u_{m_{k}}-U u_{m_{k}}\right\| .
\end{aligned}
$$

Combining (54) and the (58), we get

$$
\left\|x_{m_{k}+1}-x_{m_{k}}\right\| \longrightarrow 0, \quad \text { as } n \longrightarrow \infty \text {. }
$$

Thirdly, we show that $x_{m_{k}} \longrightarrow \bar{x}$ as $n \longrightarrow \infty$.

Using (68) and (75), we get

$$
\underset{n \longrightarrow \infty}{\limsup }\left\langle u-\bar{x}, x_{m_{k}+1}-\bar{x}\right\rangle \leq 0 .
$$

Based on $s_{m_{k}} \leq s_{m_{k}+1}, \forall k \in N$ and (46), we get

$\alpha_{m_{k}} s_{m_{k}+1}+\left(1-\alpha_{m_{k}}\right)\left(s_{m_{k}+1}-s_{m_{k}}\right) \leq 2 \alpha_{m_{k}}\left\langle u-\bar{x}, x_{m_{k}+1}-\bar{x}\right\rangle$.

So,

$$
\alpha_{m_{k}} s_{m_{k}+1} \leq 2 \alpha_{m_{k}}\left\langle u-\bar{x}, x_{m_{k}+1}-\bar{x}\right\rangle
$$

that is,

$$
s_{m_{k}+1} \leq 2\left\langle u-\bar{x}, x_{m_{k}+1}-\bar{x}\right\rangle .
$$

Taking the limit $k \longrightarrow \infty$, using (79), we obtain

$$
s_{m_{k}+1} \longrightarrow 0, \quad \text { as } k \longrightarrow \infty \text {. }
$$

Thus,

$$
s_{k} \longrightarrow 0, \quad \text { as } k \longrightarrow \infty,
$$

due to $s_{k} \leq s_{m_{k}+1}$. The proof is completed.

\section{Numerical Example}

In the section, we present a numerical experiment to demonstrate the convergence of this algorithm.

Assume $H_{1}=H_{2}=\left(R^{3},\|\cdot\|_{2}\right)$ and $T, U: R^{3} \longrightarrow R^{3}$ is defined by

$$
\begin{aligned}
& T\left(\begin{array}{l}
x \\
y \\
z
\end{array}\right)=\frac{1}{3}\left(\begin{array}{l}
x \\
y \\
z
\end{array}\right), \\
& U\left(\begin{array}{l}
a \\
b \\
c
\end{array}\right)=\left(\begin{array}{l}
0 \\
a \\
b
\end{array}\right) .
\end{aligned}
$$

Let the bounded linear operator $A$ be defined by

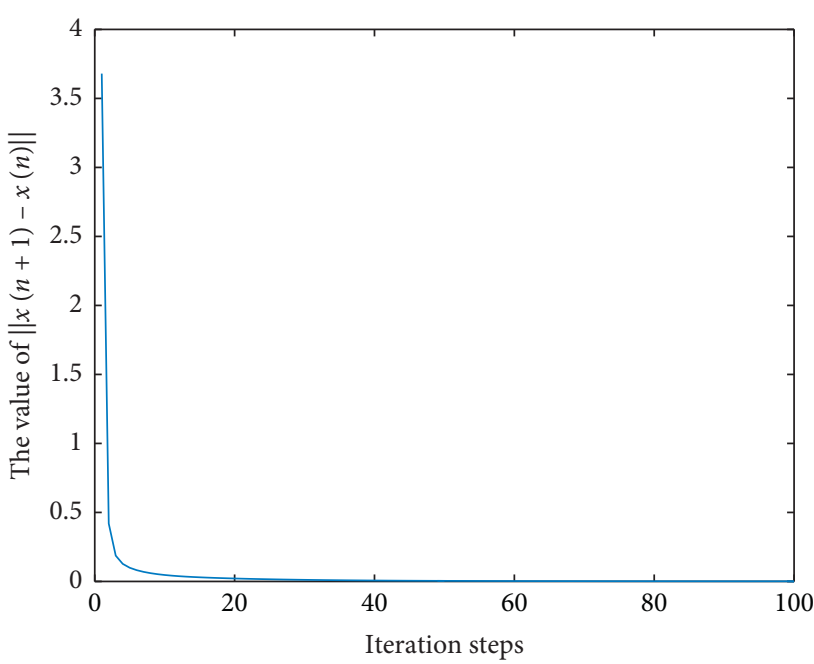

FIGURE 8: The iterative curves of algorithm (21) under different $n$.

$$
A=\left(\begin{array}{ccc}
5 & -5 & -7 \\
-4 & 2 & -4 \\
-7 & -4 & 5
\end{array}\right)
$$

Clearly, both $U$ and $T$ are 0 -demicontractive mappings. Choose the parameters as follows:

$$
\begin{aligned}
& \theta_{n}=\frac{1}{n}, \\
& \mu_{n}=\frac{1}{n}, \\
& \nu_{n}=\frac{1}{\sqrt{n}}, \quad \forall n \geq 1 .
\end{aligned}
$$

$\rho_{n}$ is chosen in the following way:

$$
\rho_{n}= \begin{cases}\frac{(1-\tau)\left\|(I-T) A x_{n}\right\|^{2}}{2\left\|A^{*}(I-T) A x_{n}\right\|^{2}}, & A x_{n} \neq T\left(A x_{n}\right), \\ 0, & \text { otherwise, }\end{cases}
$$

where $A$ is a bounded and linear mapping and $A^{*}$ is its adjoint. Then, the iterative algorithm (10) becomes as follows:

$$
\left\{\begin{array}{l}
u_{n}=x_{n}-\rho_{n} A^{*}(I-T) A x_{n}, \\
x_{n+1}=\frac{1}{n} u+\left(1-\frac{1}{n}\right)\left\{\left(1-\frac{1}{n}\right) I+\frac{1}{n} U\left[\left(1-\frac{1}{\sqrt{n}}\right) I+\frac{1}{\sqrt{n}} U\right]\right\} u_{n},
\end{array}\right.
$$

where $u=\left(\begin{array}{c}1 \\ -1 \\ 2\end{array}\right)$ is a fixed point in $R^{3}$, and the initial point $x_{1}=\left(\begin{array}{l}a_{1} \\ b_{1} \\ c_{1}\end{array}\right)=\left(\begin{array}{c}1 \\ -2 \\ 5\end{array}\right)$ and $x_{n}=\left(\begin{array}{l}a_{n} \\ b_{n} \\ c_{n}\end{array}\right)$ is generated by the algorithm (10). We plot the numbers of iterations and 


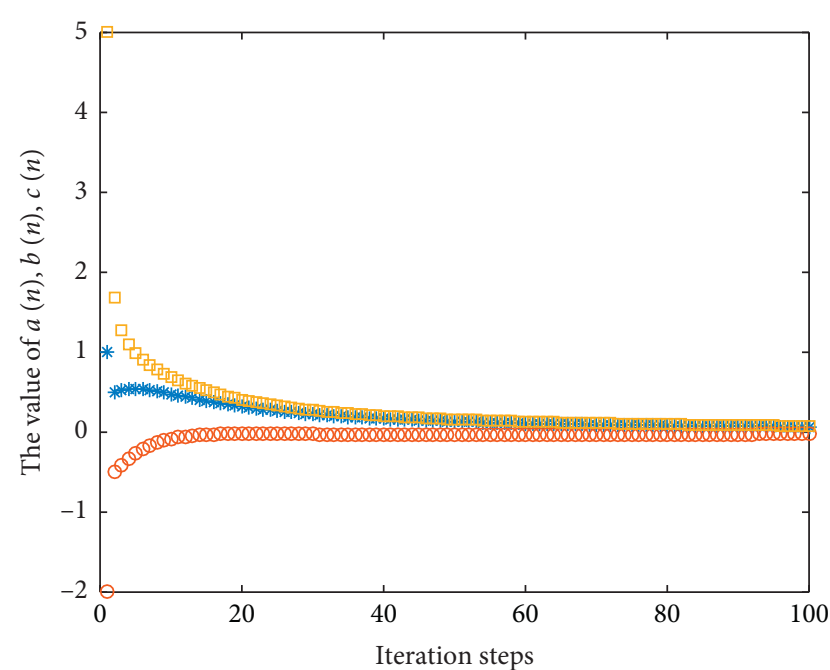

Figure 9: The iterative curves of algorithm (21) under different $n$.

$\left\|x_{n+1}-x_{n}\right\|_{2}$ in the following graphs (Figures 8 and 9), the numbers of iterations and $\left\{x_{n}\right\}=\left\{a_{n}, b_{n}, c_{n}\right\}$.

\section{Conclusion}

In this paper, we proposed a new iteration algorithm (10) and we obtained the strong convergence of the sequence $\left\{x_{n}\right\}$ for split common fixed point problems (5). The main result is an extension of the related results announced in $[15,16,27]$. The research highlights of this paper are novel algorithms and their analysis techniques. The improvement on the extension of the operator, such as the demicontractive mappings, the directed operators, the quasi-non-expansive operators, and quasi-pseudo-contractive operators will be of interest for further research in the future.

\section{Data Availability}

The data used to support the findings of this study are included within the article.

\section{Conflicts of Interest}

The authors declare that they have no conflicts of interest.

\section{Acknowledgments}

This work was supported by the Fundamental Research Funds for the Central Universities (no. JB180713), the National Science Foundation of China (no. 12031003), the 65th China Postdoctoral Science Foundation (no. 2019 M652837), and the Special Science Research Plan of the Education Bureau of Shaanxi Province of China (no. 18JK0344).

\section{References}

[1] Y. Censor and T. Elfving, "A multiprojection algorithm using Bregman projections in a product space," Numerical Algorithms, vol. 8, no. 2, pp. 221-239, 1994.
[2] C. Byrne, "Iterative oblique projection onto convex sets and the split feasibility problem," Inverse Problems, vol. 18, no. 2, pp. 441-453, 2002.

[3] X. Qin, "Smoothing algorithms for computing the projection onto a Minkowski sum of convex sets," Computational Optimization and Applications, vol. 74, no. 3, pp. 821-850, 2019.

[4] Q. An and T. Liu, "Smoothing $L_{0}$ regularization for extreme learning machine," Mathematical Problems in Engineering, vol. 2020, Article ID 9175106, , 2020.

[5] S. Y. Cho, "Generalized mixed equilibrium and fixed point problems in a Banach space," Journal of Nonlinear Sciences and Applications, vol. 09, no. 03, pp. 1083-1092, 2016.

[6] N. T. An, N. M. Nam, and X. Qin, ", "Solving k-center problems involving sets based on optimization techniques," Journal of Global Optimization, vol. 76, no. 1, pp. 189-209, 2020.

[7] Q. Fan, L. Niu, and Q. Kang, "Regression and multiclass classification using sparse extreme learning machine via smoothing group L 1/2 regularizer," IEEE Access, vol. 8, pp. 191482-191494, 2020.

[8] C. Byrne, "A unified treatment of some iterative algorithms in signal processing and image reconstruction," Inverse Problems, vol. 20, no. 1, pp. 103-120, 2004.

[9] X. Qin, L. Wang, and J. C. Yao, "Inertial splitting method for maximal monotone mappings," Journal of Nonlinear Convex Analysis, vol. 21, pp. 2325-2333, 2020.

[10] W. Takahashi, C. F. Wen, and J. C. Yao, "The shrinking projection method for a finite family of demimetric mappings with variational inequality problems in a Hilbert space," Fixed Point Theory, vol. 19, pp. 407-419, 2018.

[11] W. Takahahsi and J. C. Yao, "The split common fixed point problem for two finite families of nonlinear mappings in Hilbert spaces," Journal of Nonlinear Convex Analysis, vol. 20, pp. 173-195, 2019.

[12] A. Moudafi, "The split common fixed point problem for demicontractive mappings," Inverse Problem, vol. 26, p. 55007, 2010.

[13] L. Liu, "A hybrid steepest descent method for solving split feasibility problems involving nonexpansive mappings," Journal of Nonlinear Convex Analysis, vol. 20, pp. 471-488, 2019.

[14] Y. Censor and A. Segal, "The split common fixed point problem for directed operators," Journal of Convex Analysis, vol. 16, pp. 587-600, 2009.

[15] H. Cui and F. Wang, "Iterative methods for the split common fixed point problem in Hilbert spaces," Fixed Point Theory and Applications, vol. 2014, p. 78, 2014.

[16] O. A. Boikanyo, "A strongly convergent algorithm for the split common fixed point problem," Applied Mathematics and Computation, vol. 265, pp. 844-853, 2015.

[17] S. Y. Cho, "A convergence theorem for generalized mixed equilibrium problems and multivalued asymptotically nonexpansive mappings," Journal of Nonlinear Convex Analysis, vol. 21, pp. 1017-1026, 2020.

[18] X. Qin, S. Y. Cho, and L. Wang, "Strong convergence of an iterative algorithm involving nonlinear mappings of nonexpansive and accretive type," Optimization, vol. 67, no. 9, pp. 1377-1388, 2018.

[19] W. Takahashi, "The split feasibility problem in Banach spaces," Journal of Nonlinear Convex Analysis, vol. 15, pp. 1349-1335, 2014.

[20] L.-C. Ceng, Q. H. Ansari, and J.-C. Yao, "Relaxed extragradient methods for finding minimum-norm solutions of the split feasibility problem," Nonlinear Analysis: Theory, Methods \& Applications, vol. 75, no. 4, pp. 2116-2125, 2012. 
[21] S. Y. Cho, "A monotone Bregan projection algorithm for fixed point and equilibrium problems in a reflexive Banach space," Filomat, vol. 34, pp. 1487-1497, 2020.

[22] S. M. Alsulami and W. Takahashi, "The split common null point problem for maximal monotone mappings in Hilbert spaces and applications," Journal of Nonlinear Convex Analysis, vol. 15, pp. 793-808, 2014.

[23] X. Qin, S. Y. Cho, and L. Wang, "Iterative algorithms with errors for zero points of $\mathrm{m}$-accretive operators," Fixed Point Theory and Applications, vol. 2013, p. 148, 2013.

[24] H. He, J. Peng, and Q. Fan, "An iterative viscosity approximation method for the split common fixed-point problem," Optimization, vol. 67, 2020.

[25] T. M. Tuye, R. Promka, and P. Sunthrayuth, "Strong convergence of a generalized forwardbackward splitting method in reflexive Banach spaces," Optimization, vol. 47, 2020.

[26] X. Qin, S. Y. Cho, and L. Wang, "A regularization method for treating zero points of the sum of two monotone operators," Fixed Point Theory and Applications, vol. 2014, p. 75, 2014.

[27] Y. Yao, R. P. Agarwal, M. Postolache, and Y.-C. Liou, "Algorithms with strong convergence for the split common solution of the feasibility problem and fixed point problem," Fixed Point Theory and Applications, vol. 2014, no. 1, p. 183, 2014.

[28] K. Goebel and W. A. Kirk, Topics in Metric in Metric Fixed Point Theory, Vol. 28, Cambrige University Press, Cambridge, UK, 1990.

[29] H. K. Xu, "An iterative approach to quadratic optimization," Journal of Optimization Theory and Applications, vol. 116, no. 3, pp. 659-678, 2003.

[30] S. S. Chang, L. Wang, and L. J. Qin, "Split Equality Fixed Point Problem for Quasi-Pseudo-Contractive Mappings with Applications," Fixed point Theory and Applications, vol. 2015, p. 208, 2015.

[31] P.-E. Maingé, "Strong convergence of projected subgradient methods for nonsmooth and nonstrictly convex minimization," Set-Valued Analysis, vol. 16, no. 8, pp. 899-912, 2008. 